\title{
Reseña Bibliográfica: Colombani, M. C., Ferguson, J. y Gerardi, J. M. (Comps.) (2018). Miradas interdisciplinarias sobre el Mundo Antiguo. Encuentros de Historia, Filosofía y Literatura. Mar del Plata: Universidad Nacional de Mar del Plata. 309 pp.
}

Palabras claves: Egipto - Grecia - Roma - Estudios Interdisciplinarios Nuevas Perspectivas

Keywords: Egypt - Greece - Rome - Interdisciplinary Studies - New

Perspectives

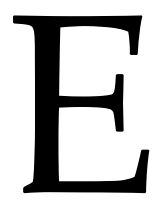

1 libro aquí reseñado es una compilación de diferentes investigaciones relativas al Mundo Antiguo. María Cecilia Colombani, Juan Ferguson y Juan

Manuel Gerardi son los encargados de presentarnos diferentes perspectivas sobre este periodo. La obra, dividida en 14 capítulos, reúne trabajos de investigadores sobre temas específicos de historia, filosofía y literatura.

En el primer apartado, Introducción, los compiladores nos presentan el detrás de escena del libro. En especial, nos adelantan la forma en que fue gestado como resultado de un intercambio académico con sede en la ciudad de Mar del Plata. A su vez, obtenemos un acercamiento a las intenciones de reproducir el espíritu de trabajo en conjunto detrás de la publicación de esta obra.

Podemos reconocer una primera división de dos capítulos pertenecientes a investigaciones sobre el Antiguo Egipto. En primer lugar, se encuentra el trabajo de Marcelo Campagno de la Universidad de Buenos Aires: “Mito, cuento, historia. A propósito de los relatos egipcios de 'La contienda entre Horus y Seth' y de 'Verdad y Mentira". Allí, el investigador realiza un acercamiento novedoso a la cuestión de la conformación del Estado. Esto lo logra mediante la revisión de ambas narraciones y de la continuidad temporal del tópico que tratan. Especialmente, se centra en las estructuras legales y cómo se produce la resolución de conflictos en las mismas. Asimismo, en el artículo, el autor estudia las diferentes instancias que caracterizan a las sociedades (tribales, estatales, etc.).

El siguiente capítulo corresponde a Juan R. G. Ferguson de la Universidad Nacional de Mar del Plata: “Trabajo especializado, división del trabajo y trabajadores durante el Reino Medio egipcio (c. 2055-1650): continuidades y rupturas en los registros de las minas del Sinaí". Este trabajo nos ofrece un acercamiento a cuestiones de la vida de los obreros en las minas. Tras la descripción general del tópico, el autor desarrolla el análisis 
sobre las continuidades y los cambios en los tipos de trabajo y de las formas de control estatal a lo largo del Imperio Medio. El foco principal del capítulo está puesto en la evolución del tipo de trabajo, el cual termina especializándose a lo largo del periodo.

Tras este recorrido por el Egipto faraónico ingresamos en el mundo griego. María Cecilia Colombani de la Universidad Nacional de Mar del Plata y de la Universidad de Morón es la autora de "La singularidad de la "versión" de Hesíodo". Colombani nos introduce en un análisis, desde la filosofía, sobre el carácter singular del pensamiento de Hesíodo y su desarrollo particular sobre el linaje divino que desarrolla en la Teogonía y en Trabajos y Días. Gracias a ello, nos deja con una nueva visión sobre un autor muy trabajado de la Antigüedad Griega. Al igual que capítulos anteriores, introduce posibles vías de investigación futura en el tema.

A continuación, Ana Iriarte de la Universidad del País Vasco nos presenta un análisis contemporáneo sobre Casandra en "Casandra o el logos des-autorizado en la Atenas democrática (y en Wikipedia)". El recorrido iconográfico, eje del capítulo, nos posibilita observar la percepción de la figura femenina mencionada desde la Antigüedad hasta la actualidad. Lo novedoso del trabajo es la recuperación de Casandra en nuestro mundo digital y, en especial, su lugar en la red Wikipedia.

Tras este estudio sobre Casandra, hallamos una aproximación a los significados y las implicancias del deporte y sus participantes en la Grecia antigua de Fábio de Souza Lessa de la Universidad Federal de Rio de Janeiro en "Competiciones deportivas en la Antigua Grecia". El autor analiza los tipos de competiciones y su significado para las ciudades. Al mismo tiempo, desarrolla el tema del deporte como una forma de expresión diferente de la violencia social.

María Regina Candido de la Universidad del Estado de Rio de Janeiro nos presenta "Las prácticas de la magia amorosa de Simeta". El centro del análisis es una mujer, Simeta, y su camino para lograr deshacer un desamor. Se trata de un acercamiento a la magia y a sus formas de reproducción y ejecución. Otro punto que cabe rescatar del trabajo es la atención que presta, en su lectura de las fuentes antiguas, al rol activo de ciertas mujeres, un tema que fue dejado de lado por muchos años y que resultaría interesante que se retomara en estudios futuros.

A continuación, Julián Gallego de la Universidad de Buenos Aires presenta "Democracia, estado, sujeto: pensar la política en la Atenas clásica". Gallego realiza un repaso sobre el significado de la palabra democracia y sus implicancias en la política de la ciudad griega. Ello lo lleva a observar el carácter situacional de la conformación del término demokratia y, por lo tanto, su fuerza parcial como referencia al poder del pueblo.

En "Otra luz para el futuro de Atenas. Eurípides, Ion", de María Fátima Silva de la Universidad de Coímbra, leemos un análisis sobre la cuestión de la localidad y la identidad ateniense. Mediante el estudio del Ion, la autora pretende encontrar el significado de la autoctonía para la identidad ateniense, tan fuerte en su periodo de máximo apogeo. Esto la lleva a resaltar tanto el rol del dios Apolo como el de Creúsa, dejando abierto a quien le corresponde el mérito de origen de la misma. 
A modo de puente entre los mundos griego y romano se encuentra el siguiente capítulo, a cargo de Alexandre Carneiro Cerqueira Lima de la Universidad Federal Fluminense, "Sympósion: entre Sophrosýne y Truphé". Allí, el autor realiza un análisis de sophrosýne y truphé en el marco del evento del sympósion y, asimismo, introduce un análisis del Otro en la figura de los aristócratas etruscos. Este capítulo nos ofrece una mirada crítica acerca de cuál era la percepción sobre los etruscos.

El siguiente capítulo escrito por Arturo R. Álvarez Hernández de la Universidad Nacional de Mar del Plata se titula “Lecturas y lectores en la poesía de Catulo". Álvarez Hernández nos introduce en el mundo editorial y de reproducción de las obras escritas en el mundo romano a partir de la obra de Catulo. Temática de gran interés y relevancia para poder entender cómo fuentes, de gran valor histórico para nosotros, vieron la luz y fueron vistos por diferentes lectores en la época de su creación.

Por su parte, Juan Manuel Gerardi, de la Universidad Nacional de Mar del Plata, nos presenta un análisis sobre eventos ampliamente descriptos del último tiempo de la Republica. En “Conflictos sociales y modalidades de la protesta popular en la crisis de la república romana", el autor problematiza el carácter de los disturbios populares. Específicamente, busca comprenderlos como elementos de la participación política de los sectores populares. Esta visión revierte la más difundida, que presenta a estos como herramientas en las manos de los grandes personajes históricos.

Continuando con la nueva luz sobre temas clásicos de la historiografía, está el trabajo de Carlos García Mac Gaw de la Universidad Nacional de la Plata y de la Universidad de Buenos Aires: "El esclavismo romano, el modo de producción antiguo y el sistema tributario. Teoría e historiografía". Allí, el autor estudia la relación entre la esclavitud, el sistema económico romano y la teoría marxista. Este capítulo ofrece un repaso sobre los debates alrededor de los modos de producción, especialmente el esclavista. García Mac Gaw finaliza el artículo presentando una nueva propuesta que involucra a los modos de producción antiguo y tributario, y su aplicación a la economía romana.

Como cierre del libro, tenemos el trabajo de Regina María da Cunha Bustamante de la Universidad Federal de Rio de Janeiro: "Espectáculos de anfiteatro en el África romana: la competición fuera de la arena". En él, analiza las representaciones de aquellos grupos que rodeaban al espectáculo, mediante lo escrito y lo figurado en un mosaico tunecino. El trabajo comprensivo de la fuente recupera grupos poco conocidos relacionados con los anfiteatros.

Esperamos haber logrado dejar de manifiesto la variedad de análisis en el libro reseñado. Una variedad conformada por varios aspectos. Primero, los periodos y los temas tratados desde las perspectivas de distintas disciplinas. Segundo, la procedencia de los autores. Encontramos una gran participación regional con autores pertenecientes a diferentes instituciones, tanto nacionales como internacionales. Este es uno de los puntos fuertes de este libro, ya que permite encontrar reunidos, en un solo espacio, los tópicos y herramientas de análisis desarrollados en diferentes Universidades. Una forma excelente de hacer conocer el lugar en que se encuentra la investigación en la región. Por 
161 | Reseña

último, pero no por ello menos importante, el libro ofrece a las nuevas generaciones de investigadores aproximaciones novedosas que les resultarán de suma utilidad cuando lleven a cabo sus propias investigaciones. Todos estos puntos que resaltamos de Miradas interdisciplinarias sobre el mundo antiguo ponen de manifiesto que las investigaciones sobre el Mundo Antiguo son un ámbito vivo y activo, desde las cuales se pueden generar nuevas ideas, pensamientos y múltiples colaboraciones.

Leandro A. Wallace

Universidad Nacional del Sur, Argentina wallace.leandro13@gmail.com

Para citar esta reseña:

Wallace, Leandro A. (2019). "Reseña Bibliográfica: Colombani, M. C., Ferguson, J. y Gerardi, J. M. (Comps.) (2018). Miradas interdisciplinarias sobre el Mundo Antiguo. Encuentros de Historia, Filosofía y Literatura. Mar del Plata: Universidad Nacional de Mar del Plata. 309 pp.". Anuario de la Escuela de Historia Virtual, 16, pp. 158-161. 